\title{
EFICÁCIA DOS ANTIDEPRESSIVOS NA FIBROMIALGIA: UMA ANÁLISE CRÍTICA A PARTIR DE DADOS RARAMENTE ENCONTRADOS NOS ARTIGOS CIENTÍFICOS
}

\author{
THE EFFECTIVENESS OF ANTIDEPRESSANT AGENTS IN \\ FIBROMYALGIA: A CRITICAL ANALYSIS OF RARELY FOUND \\ DATA IN SCIENTIFIC ARTICLES
}

\begin{abstract}
Autores: Marcelo Derbli Schafranski ${ }^{1}$, Alexandre Bueno Merlini², Ana Lúcia de Oliveira Prestes ${ }^{3}$, Bruno Ribeiro ${ }^{4}$, Juliana da Silva Geraldino ${ }^{5}$, Maria Fernanda Gauer $^{6}$

${ }^{1}$ Doutor em Medicina Interna pela UFPR. Docente de Reumatologia, Departamento de Medicina, Universidade Estadual de Ponta Grossa (UEPG). E-mail: marceloschafranski@yahoo. com.br

${ }^{2}$ Acadêmico do $5^{\circ}$ ano de Medicina da Universidade Estadual de Ponta Grossa (UEPG). E-mail: alexandre_merlini@hotmail.com

${ }^{3}$ Acadêmica do $5^{\circ}$ ano de Medicina da Universidade Estadual de Ponta Grossa (UEPG). E-mail: anadylong@hotmail.com

${ }^{4}$ Acadêmico do $5^{\circ}$ ano de Medicina da Universidade Estadual de Ponta Grossa (UEPG). E-mail: rbe.ribeiro@gmail.com

${ }^{5}$ Acadêmica do $5^{\circ}$ ano de Medicina da Universidade Estadual de Ponta Grossa (UEPG). E-mail: juliana.ge@hotmail.com

${ }^{6}$ Acadêmica do $5^{\circ}$ ano de Medicina da Universidade Estadual de Ponta Grossa (UEPG). E-mail: nanda_gauer@hotmail.com
\end{abstract}

Data de recebimento: $21 / 10 / 2013$

Data da aprovação: 01/12/2013

\section{RESUMO}

A fibromialgia (FM) corresponde a uma das doenças crônicas e incapacitantes mais prevalentes e estudadas na atualidade. Embora o número de pesquisas a seu respeito tenha crescido exponencialmente, muitos aspectos relacionados à etiologia, fisiopatologia, genética e terapêutica ainda permanecem incertos. Estima-se que a prevalência é de aproximadamente $2 \%$ na população em geral, acometendo de $7,4 \%$ a $10 \%$ de mulheres entre 40 a 60 anos. Embora sintomas como dor difusa, fadiga, distúrbios do sono sejam clássicos, a FM abrange um amplo espectro de manifestações clínicas, inclusive doenças neuropsiquiátricas, como a depressão. A concomitância deste distúrbio de humor é presente em $26,8 \%$ a $70 \%$ dos pacientes fibromiálgicos. Nesse contexto, o objetivo do presente estudo é analisar a eficácia dos antidepressivos no tratamento da FM. Foram analisados os ensaios clínicos randomizados e controlados (RCTs) existentes, utilizando as variáveis NNT (number needed to treat) / NNH (number needed to harm), e tamanho de efeito por meio do coeficiente $d$ de Cohen. Os cálculos envolveram questionários e escalas já validados na avaliação da fibromialgia. Apenas três RCTs foram encontrados, envolvendo os seguintes fármacos: duloxetina $(\mathrm{NNT}=7,9 ; \mathrm{d}=3,11)$; milnaciprano $(\mathrm{NNT}=6,3 ; \mathrm{d}=$ $-5,87)$; moclobemida $(\mathrm{NNT}=30,0 ; \mathrm{d}=-0,15)$ e amitriptilina $(\mathrm{NNT}=4,0 ; \mathrm{d}=-0,05)$. A partir da análise destas variáveis dificilmente encontradas em artigos científicos, notou-se a necessidade de que mais RCTs sejam executados, e de que os pesquisadores demonstrem estes índices, a fim de que a utilização de tratamentos pouco eficazes e dispendiosos em pacientes acometidos por esta incômoda síndrome seja evitada.

Palavras-chave: Fibromialgia. Antidepressivos. NNT. NNH. 


\begin{abstract}
Fibromyalgia (FM) corresponds to one of the most prevalent and studied chronic and disabling disorder currently. Although the number of its researches has grown exponentially, many aspects related to its etiology, pathophysiology, genetics and therapy remain uncertain. The estimated prevalence is about $2 \%$ in general population, reaching $7.4 \%$ at? $10 \%$ of women with 40 to 60 years. Even though symptoms such as diffuse pain, fatigue and sleep disturbances are classic, it covers a broad spectrum of clinical manifestations, including neuropsychiatric disorders, like depression. The concomitance of this mood disorder is present in up to $26.8 \%$ at? $70 \%$ of patients with fibromyalgia. Thus, the objective of this study is to analyze the antidepressants efficacy in the treatment of FM. We analyzed the randomized controlled trials (RCTs) existing, using variables as NNT (number needed to treat) / NNH (number needed to harm) and size effect - Cohen's $d$. The estimates involved questionnaires and scales already validated on the evaluation of fibromyalgia.Only three RCTs were found, involving the following drugs: duloxetine ( $\mathrm{NNT}=7,9 ; \mathrm{d}=3,11)$; milnacipran $(\mathrm{NNT}=6,3 ; \mathrm{d}=$ $-5,87)$; moclobemide ( $N N T=30,0 ; d=-0,15)$ and amitriptyline $(N N T=4,0 ; d=-0,05)$. Through these variables difficultly found in scientific articles, it was noted the need for more RCTs are implemented, and for the studiers show these rates; so the use of expensive and less effective in patients affected by this troublesome syndrome can be avoided.
\end{abstract}

Keywords: Fibromyalgia. Antidepressant agents. NNT. NNH.

\section{Introdução}

Fibromialgia (FM) é uma doença prevalente, crônica e incapacitante, de etiologia ainda desconhecida. É caracterizada por dor generalizada, sensibilidade difusa e amplo espectro de outros sintomas. Considera-se também a existência de pontos sensíveis (tender points) - locais dolorosos quando uma força de $4 \mathrm{~kg} / \mathrm{A}$ é aplicada pela palpação digital - pelo corpo (SMITH et al., 2011).

Tecnicamente, pode ser definida pelo histórico de dor generalizada por pelo menos três meses, aliada à existência de dor em pelo menos 11 de 18 pontos sensíveis.

As manifestações não são homogêneas, tornando difícil o seu diagnóstico. A FM apresenta proporções variáveis de ansiedade e depressão como comorbidades, que dependem das características psicológicas de cada paciente. Portanto, a avaliação médica deve incluir não só a presença de dor generalizada ou pontos sensíveis, mas também a existência de distúrbios de humor (KÖLLNER et al., 2011).

Alguns estudos relataram que os pacientes com FM demonstram altos níveis de alexitimia e raiva, que, associados à depressão, estresse no trabalho e traumas durante a infância, são contribuintes para a sua etiologia e, por isso, os antidepressivos podem constituir um eficaz manejo terapêutico. Por outro lado, pesquisadores identificaram a influência do suporte social e do contexto emocional sob o limiar da dor.

Estes e outros resultados levaram à conclusão de que existe uma ligação intrínseca entres transtornos depressivos e FM. Outros estudiosos classificaram-nas numa mesma ampla categoria de distúrbios relacionados ao estresse (ARNOLD et al., 2011). Por fim, a depressão é uma frequente (presente em 28,6\% a $70 \%$ dos pacientes) comorbidade associada à fibromialgia, agravando a história natural desta doença (KASSAM et al, 2006).

\section{Perspectiva histórica}

Infelizmente, até poucas décadas atrás, a fibromialgia era considerada por muitos médicos e pesquisadores como um distúrbio psicológico. Somente no século XX foi finalmente reconhecida como uma condição física real.

Os maiores eventos no estudo da FM começam com Hipócrates, que descreveu uma síndrome com 
placas de maior consistência em músculos de pacientes com artrite reumatoide. No início do século XX, Ralph Stockman reportou a existência de nódulos com extrema sensibilidade à pressão nos pacientes com FM e a biópsia revelou hiperplasia inflamatória nessas áreas (MARSON et al., 2008).

Na década de 1970, foi descrita a presença de distúrbios do sono e também o papel dos neurotransmissores (serotonina e substância P) em sua fisiopatologia; foi reportada, ainda, por Yunus, a concomitância com síndromes somáticas: intestino irritável, cefaleias e distúrbios psiquiátricos. Em 1976, Philip Kahler Hench substitui o termo "fibrosite", usado até então, por "fibromialgia" (HUDSON et al., 1985).

Em 1981, Muhammad B. Yunus e Alphonse T. Mais realizaram o primeiro ensaio controlado que levou à validação dos sintomas conhecidos e dos tender points. O termo "fibromialgia" se torna então universalmente aceito. E já em 1987 a American Medical Association (AMA) passa a considerar a FM como uma condição física real.

Em 1990, o American College of Rheumatology (ACR) desenvolveu critérios diagnósticos, e a participação do mecanismo neuro-hormonal com a sensibilização central foi estabelecida. E em 2007, a Food and Drug Administration (FDA) aprovou a primeira droga para o seu tratamento (MARSON et al., 2008). Atualmente, inúmeras pesquisas continuam a investigar a etiopatogênese e formas de tratamento dessa síndrome.

\section{Epidemiologia}

De acordo com estudos epidemiológicos, a FM afeta cinco milhões de americanos com 18 anos ou mais, sendo $80 \%$ a $90 \%$ mulheres. Os diagnósticos, em sua maioria, são estabelecidos em idade intermediária, mas os sintomas podem aparecer mais cedo (CAVALCANTE et al., 2006).

A prevalência na população em geral é de $2 \%$, mas aumenta com a idade, sendo maior em mulheres da quinta à sétima década (7.4\% a 10\%) (RUSSELL et al., 2008).

Estudos recentes indicam a FM como a terceira doença reumática mais comum, atrás de osteoartrite e do lumbago. A distribuição da doença por sexo não muda nas crianças. Dos casos vistos em clínicas reumatológicas, $15 \%$ são atribuídos à FM, e em outras especialidades são $6 \%$, mostrando o incrível aumento de sua incidência (SHUSTER et al., 2009).

\section{Fatores de risco}

Devido às características multifatoriais que compõem a FM, a determinação de seus fatores de risco se torna um estudo árduo. Alguns estudos associam seu desenvolvimento a um estresse físico, ou emocional ou a eventos traumáticos. Outros acreditam na sucessão de injúrias repetitivas, ligadas a outra doença, ou acreditam que ocorra espontaneamente. Vários estudos envolvem o processamento da dor feito pelo sistema nervoso central e o papel regulador dos genes na detecção dos estímulos dolorosos. $\mathrm{Na}$ presença de história familiar positiva, as chances do desenvolvimento de FM aumentam, tendo a hereditariedade e os fatores ambientais como possíveis responsáveis.

Alguns dos fatores de risco mais bem descritos são: depressão (hospitalização, tratamento, histórico familiar); níveis elevados de somatização e ansiedade; sensação de derrame articular; parestesia e rigidez matinal; distúrbios do sono; intestino irritável; má percepção ou satisfação do estado de saúde; eventos traumáticos; doenças febris; gênero, sendo sete vezes mais frequente em mulheres; idade; história familiar (WOLFE et al., 1995).

\section{Manifestações clínicas}

Em 1990, o American College of Rheumatology (ACR) estabeleceu critérios diagnósticos à síndrome da fibromialgia, e assim ela era diagnosticada até há pouco tempo. (CLAUW et al., 2011). Estes critérios consistiam na avaliação da dor generalizada em combinação com a dor à palpação em pelo menos 11 dos 18 pontos específicos estabelecidos (tender points) (WOLFE et al., 1990).

Recentemente, em 2010, o ACR propôs um método de critério diagnóstico alternativo para a FM, ou seja, este não requer a presença de pontos sensíveis, mas inclui uma lista de outros sintomas, considerando-se a fadiga, o sono não reparador $\mathrm{e}$ sintomas cognitivos como os mais importantes. (WOLFE et al., 2011). Ainda existem alguns outros sintomas que podem ser incluídos, como cefaleia, depressão, dores e cólicas abdominais (YUNUS, 2007). (Ver Tabela 01). 
Tabela 1 - Principais sintomas da fibromialgia

\begin{tabular}{c|l}
\hline SINTOMA & \multicolumn{1}{c}{ DESCRIÇÃO } \\
\hline Distúrbios do sono & $\begin{array}{l}\text { Ocorre em até } 70 \% \text { a } 75 \% \text { dos pacientes com FM. Fadiga matinal, que é o melhor indicador para } \\
\text { sono não reparador está presente em } 75 \% \text { a } 80 \% \text { dos pacientes. Sono não reparador, ou andar se } \\
\text { sentindo cansado são reclamações comuns. }\end{array}$ \\
\hline Fadiga & $\begin{array}{l}\text { Fadiga pode ocorrer em } 80 \% \text { a } 90 \% \text { dos pacientes com fibromialgia e é geralmente caracterizada } \\
\text { pela exaustão, falta de energia, e um sentimento de fraqueza generalizada. Fadiga ou exaustão } \\
\text { pode ser, para alguns pacientes, mais difícil de lidar do que a dor generalizada. }\end{array}$ \\
\hline Cefaléia & $\begin{array}{l}\text { Cefaléias tensionais e enxaquecas são presentes em mais de 50\% dos pacientes com FM. A } \\
\text { enxaqueca está associada com um aumento da resposta à dor e hiperalgesia a vários estímulos } \\
\text { como pressão mecânica, calor, frio, som e luz. Sensibilização central pode ser uma via comum } \\
\text { para dores de cabeça em pacientes com FM. }\end{array}$ \\
\hline Doenças psiquiátricas & $\begin{array}{l}\text { Muitos estudos têm mostrado que a fibromialgia está fortemente associada com sintomas } \\
\text { depressivos e à ansiedade, e também com histórico pessoal ou familiar de depressão e } \\
\text { acompanhamento por tratamento por antidepressivos. A FM está associada com uma alta e } \\
\text { significante taxa de transtornos de humor e ansiedade e sintomas somáticos comparados com } \\
\text { pacientes que possuem outras condições de dor crônica, como artrite reumatóide. }\end{array}$ \\
\hline
\end{tabular}

O diagnóstico de FM requer estes sintomas. Mesmo assim, o paciente deve ter alguns dos outros sintomas que também são comuns, em busca de alcançar um escore composto que conduzirá a um diagnóstico.

Alguns estudos experimentais têm identificado um grande número de outras anormalidades em pacientes com FM, incluindo aumento da sensibilidade a vários tipos de estímulos dolorosos, aumento da sensibilidade a outros estímulos sensoriais e alterações em mecanismos modulatórios da dor. A qualidade de vida em fibromiálgicos pode ser comprometida significativamente, a doença pode interferir na habilidade laboral e no desempenho em atividades regulares diárias (YUNUS, 2007).

\section{Fisiopatologia}

Pesquisadores têm acreditado que a fisiopatologia da fibromialgia é devida a mecanismos anormais dos centros da dor. (ABELES et al., 2007). Parece haver um grande número de processos do sistema nervoso central no cérebro e na medula que acusam anormalidades em pacientes com FM (CEKO et al., 2012).

\section{Percepção da dor alterada em pacientes com FM}

Medidas subjetivas da dor, avaliações objetivas de reflexos periféricos da dor e estudos de imagens cerebrais têm indicado que pacientes com FM percebem a dor diferentemente dos indivíduos saudáveis (GRACELY et al., 2002). Esses pacientes têm fisiologicamente limiares da dor mais baixos. Existem ainda diferenças no processamento da dor. O limiar em que estímulos normais (pressão, calor e frio) se tornam dolorosos é mais baixo em pacientes com FM (WOOD et al., 2007). Na verdade, muitas evidências indicam que pontos dolorosos são apenas locais mais sensíveis à dor por pressão em todos os indivíduos e que paciente com FM tem uma sensibilidade à pressão aumentada em pontos não dolorosos também. Há alguns estudos que mostram que pacientes com FM têm a sensibilidade aumentada a muitos tipos de estímulos dolorosos, incluindo a pressão em pontos não dolorosos, calor, frio, estímulos elétricos e a injeção intramuscular de solução salina hipertônica (BERGLUND et al., 2002).

\section{Sensibilização central}

A sensibilização central consiste no aumento da excitabilidade de neurônios da medula que 
transmitem a informação da dor para o córtex pelas fibras C. Essa sensibilização parece ser o principal fator que contribui para a hiperalgesia. É associada à atividade nervosa espontânea, campos receptivos expandidos e aumento de resposta a estímulos no interior da medula espinhal. Somação temporal anormal (wind-up) é o fenômeno pelo qual, após um período inicial de estímulos dolorosos subsequentes, estímulos iguais são percebidos como mais intensos. Essa aumentada "segunda dor", que ocorre em todos, é exagerada em pacientes com fibromialgia (STAUD et al., 2001).

Esta resposta aumentada pode ser relacionada a um ou mais de inúmeros fatores: (1) uma fonte periférica de entrada contínua de nociceptores-C não originados pelo estímulo aplicado; (2) receptores de ácido N-metil-D-aspártico (NMDA) sensibilizados em neurônios nociceptivos centrais; (3) anormalidades na modulação descendente; e (4) processamento anormal em níveis supraespinais (CEKO et al., 2012).

Apesar do fato de que as específicas anormalidades que levam à excitabilidade aumentada desses neurônios não sejam conhecidas, evidências crescentes indicam que os pacientes com FM sentem uma amplificação anormal da dor (STAUD et al., 2001).

\section{Neurotransmissores}

A serotonina é um neurotransmissor produzido por neurônios no tronco cerebral e é derivado do triptofano. Apresenta alguns efeitos inibitórios em diversas vias da dor e é também amplamente distribuído. Serotonina aumentada no cérebro leva a uma atenuação da sinalização da dor e diminuição da liberação da substância P na medula espinhal (STAUD et al., 2001).

\section{Diagnóstico}

Apesar do avanço da medicina diagnóstica, a identificação da fibromialgia ocorre em $75 \%$ dos pacientes afetados, e quando ela é feita, tem uma duração média de cinco anos até que se alcance o diagnóstico (ARNOLD et al., 2011).

Como em outros transtornos, a anamnese e o exame físico completos são necessários para estabelecer o diagnóstico, sendo que exames laboratoriais e radiológicos são necessários apenas na presença de comorbidades ou para afastar outras possibilidades (MARTINS et al., 2012).
Ao avaliar as articulações, a presença de edema, crepitação, sensibilidade (alodinia ou hiperalgesia) ou alterações de movimento devem ser relatadas, bem como fraqueza ou dor anormal de origem periférica (ARNOLD et al., 2011).

$\mathrm{O}$ relato de dor generalizada por pelo menos três meses e a existência de dor em pelo menos 11 dos 18 pontos sensíveis, os chamados tender points, como explicado anteriormente, foram pela primeira vez definidos pelo ACR, em 1990, como os critérios diagnósticos para fibromialgia (BOOMERSHINE, 2012).

A concepção dos pontos sensíveis segue o Manual Tender Point Survey (MTPS), uma classificação de aumentos da sensibilidade e da especificidade diagnósticas baseada em:

a) Localização

b) Posicionamento do paciente e do examinador

c) Técnica de pressão

d) Escores de gravidade: 0 (ausência de dor) - 10 (dor insuportável); um escore de 2 pontos caracteriza um ponto sensível como positivo. (OKIFUJI et al., 1997).

Posteriormente, Wolf e colaboradores criaram um novo modelo de critérios, que não envolve a presença de pontos dolorosos, mas, sim, a existência de sintomas somáticos (GEISSER et al., 2013).

\section{Considerações genéticas}

Tendo em vista a presença de vários casos em uma mesma família, o papel dos genes na fisiopatologia da fibromialgia tem sido discutido há décadas, sendo que os primeiros estudos desenvolvidos foram realizados em famílias que apresentavam membros com sintomatologia semelhante, o que foi somado depois à utilização da genotipagem.

No entanto, os estudos ainda não demonstraram resultados concretos. Háevidências que polimorfismos não específicos em circuitos serotoninérgicos, dopaminérgicos, ou ainda catecolaminérgicos, estejam ligados à etiopatogenia da doença (MATSUDA et al., 2010). Recentemente, Mergener relatou um provável efeito do SNPT102C no gene do receptor HTR2A no metabolismo da 5-HT, mas ainda faltam estudos para explicar todos os genes envolvidos na fisiopatologia da doença (MERGENER et al., 2011). 


\section{Tratamento}

\section{Tratamento não farmacológico}

A terapia multidisciplinar é de importância inquestionável na fibromialgia. O tratamento da doença é uma aliança entre tratamento farmacológico, mudança de estilo de vida e tratamento não farmacológico (MARTINS et al., 2012). É papel do médico estabelecer uma boa relação médico-paciente, o que ajuda no controle da doença e diminui chances de abandono da terapia. Por conta disso, o médico também deve encorajar o paciente a efetuar mudanças de hábitos e fazê-lo prosseguir com o tratamento não farmacológico, defendendo a eficácia deste. As medidas incluem tratamento precoce das infecções, exercício aeróbico ou anaeróbico, conforme prescrito pelo médico, alongamento, sono saudável, evitar oscilações de humor, tratamento de depressão e ansiedade, acupuntura, fitoterapia, terapia de grupo e, se necessário, aconselhamento psiquiátrico, entre outros (GOLDENBERG, 2008).

Existem vários estudos indicando os benefícios de uma infinidade de formas de manejo não farmacológico da fibromialgia, tais como: hidroterapia, fisioterapia, acupuntura, terapia cognitivo-comportamental, entre outros, alguns deles indicados como adjuvantes, a fim de melhorar a qualidade de vida dos pacientes (BUSCH et al., 2007).

Segundo Gualano et al., a atividade física tem efeito anti-inflamatório não só na fibromialgia, mas também em outras doenças crônicas. Desta forma, o paciente é beneficiado através da diminuição da quantidade e doses de remédios (THIEME et al., 2006).

\section{Tratamento farmacológico}

O tratamento farmacológico da fibromialgia inclui, entre outros: antidepressivos, que diminuem a dor e melhoram a qualidade de vida do paciente; analgésicos, que reduzem a dor e como os antidepressivos, melhoram a qualidade de vida do paciente; ansiolíticos, úteis no tratamento da ansiedade, também proporcionando uma melhor qualidade de sono; relaxantes musculares, que aliviam a tensão muscular e a dor crônica; anticonvulsivantes, utilizados no manejo da dor crônica de pacientes deprimidos e pacientes com distúrbios do sono. (Ver Tabelas 02, 03 e 04).

\section{Outros}

- O pramipexol é um agonista dopaminérgico utilizado no tratamento da doença de Parkinson, o qual, apesar de não ter efeito sobre a dor, melhora a qualidade do sono em pacientes com fibromialgia (HÄUSER et al., 2009).

- A tizanidina é um agonista alfa-2-adrenérgico de ação central que tem efeito semelhante aos relaxantes musculares em pacientes com fibromialgia (SMITH et al., 2011).

\section{A eficácia dos antidepressivos}

Baseado em revisão da literatura médica, por meio de plataformas como PubMed, Cochrane, Lilacs e Medline, foi proposto como objetivo deste estudo a avaliação dos agentes antidepressivos no tratamento da fibromialgia e, subsequentemente, sobre os sintomas depressivos dos pacientes acometidos.

Apenas ensaios clínicos randomizados e controlados (RCT's) foram utilizados, diminuindo significativamente o número de estudos abordados.

Além das drogas antidepressivas, RCT's envolvendo drogas de outras classes farmacológicas também foram encontrados, como: gabapentina, naltrexona, pramipexol, pregabalina, piridostigmina, oxibato sódico, terguride e dolasetron.

Dos estudos relativos aos antidepressivos, foram encontrados três que envolviam os seguintes fármacos: (1) duloxetina, inibidor seletivo da recaptação da serotonina (ARNOLD et al., 2004); (2) milnacipram, inibidor não seletivo da recaptação da serotonina e da noradrenalina (ARNOLD et al., 2010); e (3) moclobemida, inibidor da monoaminoxidase (MAO) e amitriptilina, um antidepressivo tricíclico

$\mathrm{O}$ número de pacientes em cada grupo (tratamento com antidepressivo $\mathrm{x}$ controle com placebo), os desfechos primários e secundários foram coletados, bem como o NNT (number needed to treat), $\mathrm{NNH}$ (number needed to harm), e o tamanho de efeito, por meio do coeficiente $d$ de Cohen, foram calculados. (ver Tabela 5). 
Tabela 2 - Analgésicos e antidepressivos utilizados no tratamento da fibromialgia

\begin{tabular}{|c|c|c|c|c|}
\hline \multicolumn{5}{|c|}{ Analgésico } \\
\hline & Mecanismo de Ação & Metabolismo & Excreção & $\begin{array}{l}\text { Principais } \\
\text { Reações Adversas }\end{array}$ \\
\hline Tramadol & $\begin{array}{l}\text { Liga-se a receptores no sistema } \\
\text { nervoso central (SNC), inibe vias } \\
\text { ascendentes da dor e a recaptação } \\
\text { de noradrenalina e serotonina }\end{array}$ & Metabolismo hepático & Urina & $\begin{array}{l}\text { Rubor, prurido, constipação, } \\
\text { náuseas, insônia, fraqueza, tontura, } \\
\text { cefaléia, sonolência }\end{array}$ \\
\hline \multicolumn{5}{|c|}{$\begin{array}{ll}\text { Antidepressivos } \\
\end{array}$} \\
\hline & Mecanismo de Ação & Metabolismo & Excreção & Principais Reações Adversas \\
\hline Amitriptilina & $\begin{array}{l}\text { Aumenta a concentração } \\
\text { sináptica de serotonina e } \\
\text { noradrenalina no SNC pela } \\
\text { inibição da recaptação dos } \\
\text { mesmos. }\end{array}$ & $\begin{array}{l}\text { Metabolismo hepático } \\
\text { por CYP450, 2C19, } \\
\text { 1A2 e 2D6. }\end{array}$ & Urina e fezes & $\begin{array}{l}\text { Visão turva, constipação, diarréia, } \\
\text { tontura, boca seca, cefaléia, perda } \\
\text { de apetite, náuseas, desordens do } \\
\text { sono, fraqueza }\end{array}$ \\
\hline Duloxetina & $\begin{array}{l}\text { Inibe a recaptação neuronal } \\
\text { de serotonina e noradrenalina; } \\
\text { inibidor fraco da recaptação de } \\
\text { dopamina. }\end{array}$ & $\begin{array}{l}\text { Metabolismo hepático } \\
\text { pelo CYP1A2 e } 2 \text { D6 }\end{array}$ & Urina e fezes & $\begin{array}{l}\text { Náuseas, boca seca, dor de cabeça, } \\
\text { constipação, desordens do sono }\end{array}$ \\
\hline Milnaciprano & $\begin{array}{l}\text { Inibe a recaptação de } \\
\text { noradrenalina e serotonina, } \\
\text { especialmente a primeira. }\end{array}$ & Conjugação & Urina & $\begin{array}{l}\text { Náuseas, cefaléia, constipação, } \\
\text { insônia }\end{array}$ \\
\hline Venlafaxina & $\begin{array}{l}\text { Inibe a recaptação neuronal } \\
\text { de serotonina e noradrenalina; } \\
\text { inibidor fraco da recaptação de } \\
\text { dopamina. }\end{array}$ & $\begin{array}{l}\text { Metabolismo hepático } \\
\text { pela CYP2D6 }\end{array}$ & Urina & $\begin{array}{l}\text { Cefaléia, insônia, náusea, astenia, } \\
\text { tontura, alterações na ejaculação, } \\
\text { sonolência, boca seca, sudorese }\end{array}$ \\
\hline Desvenlafaxina & $\begin{array}{l}\text { Inibe a recaptação neuronal de } \\
\text { serotonina e noradrenalina. }\end{array}$ & Conjugação & Urina & $\begin{array}{l}\text { Náuseas, cefaléia, tontura, boca } \\
\text { seca, hiperidrose, diarréia, } \\
\text { constipação }\end{array}$ \\
\hline
\end{tabular}

Tabela 3 - Ansiolíticos utilizados no tratamento da fibromialgia

\begin{tabular}{|c|c|c|c|c|}
\hline \multicolumn{5}{|c|}{ Ansiolítico } \\
\hline & Mecanismo de Ação & Metabolismo & Excreção & $\begin{array}{l}\text { Principais } \\
\text { Reações Adversas }\end{array}$ \\
\hline Alprazolam & $\begin{array}{l}\text { Liga-se aos receptores } \\
\text { benzodiazepínicos no neurônio } \\
\text { pós-sináptico GABA no SNC. O } \\
\text { efeito inibitório do GABA é devido } \\
\text { ao aumento da permeabilidade da } \\
\text { membrana neuronal aos íons cloreto, } \\
\text { gerando hiperpolarização. }\end{array}$ & $\begin{array}{l}\text { Metabolismo hepático } \\
\text { pelo CYP3A4 }\end{array}$ & Urina & $\begin{array}{l}\text { Aumento ou diminui-ção do } \\
\text { apetite, sedação, constipa-ção, } \\
\text { ganho ou perda de peso, fadiga, } \\
\text { sonolência, boca seca, } \\
\text { disartria, diminuição } \\
\text { da memória,depressão, } \\
\text { irritabilidade }\end{array}$ \\
\hline Clonazepam & $\begin{array}{l}\text { Deprime a transmissão nervosa no } \\
\text { córtex motor }\end{array}$ & Metabolismo hepático & Urina & Sonolência é a mais comum \\
\hline Zolpidem & $\begin{array}{l}\text { Afinidade seletiva pelo alfa-1 } \\
\text { do receptor ômega-1 do GABA. } \\
\text { Aumenta a condutância GABAérgica } \\
\text { de cloreto, hiperpolarizando } \\
\text { membranas neuronais e reduzindo a } \\
\text { atividade excitatória. }\end{array}$ & $\begin{array}{l}\text { Metabolismo hepático } \\
\text { principalmente pela } \\
\text { CYP3A4 }\end{array}$ & $\begin{array}{l}\text { Urina, bile e } \\
\text { fezes }\end{array}$ & Cefaléia, tontura, sonolência \\
\hline Zaleplon & $\begin{array}{l}\text { Interage com o receptor } \\
\text { GABA ligando-se ao receptor } \\
\text { benzodiazepínico ômega-1. }\end{array}$ & $\begin{array}{l}\text { Metabolismo } \\
\text { principalmente por } \\
\text { oxidação do aldeído } \\
\text { e em menor grau via } \\
\text { CYP3A4 }\end{array}$ & Urina & Cefaléia é a mais comum \\
\hline
\end{tabular}


conclusão

\begin{tabular}{|c|c|c|c|c|}
\hline \multicolumn{5}{|c|}{ Ansiolítico } \\
\hline Trazodona & $\begin{array}{l}\text { Inibe a recaptação de serotonina, } \\
\text { induz mudanças nos adrenoceptores } \\
\text { pré-sinápticos de 5-HT diminuindo } \\
\text { a sensibilidade dos mesmos. } \\
\text { Também bloqueia receptores de } \\
\text { histamina (H1) e receptores alfa-1- } \\
\text { adrenérgicos. }\end{array}$ & $\begin{array}{l}\text { Metabolismo hepático } \\
\text { pelo CYP3A4 }\end{array}$ & Urina e fezes & $\begin{array}{l}\text { Sedação, náuseas, cefaléia, boca } \\
\text { seca, visão turva, tonturas }\end{array}$ \\
\hline Buspirona & $\begin{array}{l}\text { Tem uma elevada afinidade pelos } \\
\text { receptores serotoninérgicos 5-HT1A } \\
\text { e 5-HT2 e tem afinidade moderada } \\
\text { por receptores de dopamina D2. }\end{array}$ & $\begin{array}{l}\text { Metabolismo hepático } \\
\text { por oxidação }\end{array}$ & Urina e fezes & Tontura é a mais comum \\
\hline Temazepam & $\begin{array}{l}\text { Liga-se aos receptores } \\
\text { benzodiazepínicos no neurônio } \\
\text { GABA pós-sináptico no SNC. O } \\
\text { efeito inibitório do GABA é devido } \\
\text { ao aumento da permeabilidade da } \\
\text { membrana neuronal aos íons cloreto, } \\
\text { gerando hiperpolarização. }\end{array}$ & Metabolismo hepático & Urina & $\begin{array}{l}\text { Sonolência,euforia, fraqueza, } \\
\text { confusão, diarréia, tontura, } \\
\text { letargia, vertigem }\end{array}$ \\
\hline Oxibato de sódio & $\begin{array}{l}\text { Derivado do GABA, atua através } \\
\text { de receptores específicos gama- } \\
\text { hidroxibutirato (GHB) e GABA (B). }\end{array}$ & $\begin{array}{l}\text { Metabolismo através } \\
\text { do Ciclo de Krebs e } \\
\text { beta-oxidação }\end{array}$ & $\begin{array}{l}\text { Pulmonar e } \\
\text { urina }\end{array}$ & $\begin{array}{l}\text { Cefaléia, náuseas, tontura, dor, } \\
\text { faringite, infecção, sonolência }\end{array}$ \\
\hline
\end{tabular}

Tabela 4 - Anticonvulsivantes e relaxantes musculares utilizados no tratamento da fibromialgia

\begin{tabular}{l|l|l|l|l}
\hline & Mecanismo de Ação & Metabolismo & Excreção & $\begin{array}{l}\text { Principais Reações } \\
\text { Adversas }\end{array}$ \\
\hline Pregabalina & $\begin{array}{l}\text { Liga-se à subunidade alfa2-delta } \\
\text { dos canais de cálcio inibindo a } \\
\text { liberação do neurotransmissor } \\
\text { excitatório. }\end{array}$ & Metabolismo insignificante & Urina & $\begin{array}{l}\text { Tremores, ganho de peso, } \\
\text { sonolência, ataxia, } \\
\text { edema, tonturas }\end{array}$ \\
\hline Gabapentina & $\begin{array}{l}\text { Liga-se à subunidade alfa2- } \\
\text { delta dos canais de cálcio, é } \\
\text { estruturalmente semelhante } \\
\text { à pregabalina, mas não foi } \\
\text { aprovada para tratamento. }\end{array}$ & Metabolismo insignificante & Urina & $\begin{array}{l}\text { Tonturas, ataxia, fadiga, } \\
\text { infecção viral, sonolência }\end{array}$ \\
\hline \multirow{2}{*}{ Tiagabina } & $\begin{array}{l}\text { Aumenta a atividade do } \\
\text { GABA, através da ligação ao } \\
\text { receptor deste.Inibe a captação } \\
\text { nos neurônios pré-sinápticos, } \\
\text { aumentando a disponibilidade de } \\
\text { GABA. }\end{array}$ & $\begin{array}{l}\text { Metabolismo hepático pelo } \\
\text { CYP (principalmente 3A4) }\end{array}$ & $\begin{array}{l}\text { urina e } \\
\text { como droga } \\
\text { inalterada }\end{array}$ & $\begin{array}{l}\text { Náuseas, fraqueza, tremores, } \\
\text { tonturas, nervosismo,dimi- } \\
\text { nuiço da concentração, } \\
\text { sonolência }\end{array}$ \\
\hline
\end{tabular}

\begin{tabular}{l|l|l|l|l}
\hline \multicolumn{3}{c}{ Muscle Relaxants Relaxantes Musculares } \\
\hline & Mecanismo de Ação & Metabolismo & Excreção & $\begin{array}{l}\text { Principais } \\
\text { Reações Adversas }\end{array}$ \\
\hline Ciclobenzaprina & $\begin{array}{l}\text { Relaxante muscular de ação } \\
\text { central que reduz a atividade } \\
\text { motora somática pela ação sobre } \\
\text { os neurônios motores alfa e } \\
\text { gama. }\end{array}$ & $\begin{array}{l}\text { Metabolismo hepático pelo } \\
\text { CYP3A4, 1A2 e 2D6 }\end{array}$ & Urina e fezes & $\begin{array}{l}\text { Boca seca, tontura, } \\
\text { sonolência }\end{array}$ \\
\hline
\end{tabular}


Tabela 5 - Eficácia dos antidepressivos na fibromialgia, baseado em RCT's

\begin{tabular}{|c|c|c|c|c|c|}
\hline Artigo & $\begin{array}{l}\text { Desfecho } \\
\text { Primário }\end{array}$ & $\begin{array}{l}\text { Desfechos } \\
\text { Secundários }\end{array}$ & NNT / NNH & $\begin{array}{l}\text { Tamanho de } \\
\text { efeito }\end{array}$ & Melhora da Depressão \\
\hline $\begin{array}{c}\text { A double-blinded, } \\
\text { multicenter trial } \\
\text { comparing duloxetine } \\
\text { with placebo in } \\
\text { the treatment of } \\
\text { fibromyalgia patients } \\
\text { with or without major } \\
\text { depressive disorder } \\
\text { Arthritis \& Reumatism } \\
2004 \\
\text { Arnold LM } \text { et al. } \\
\text { Número de patientes: } \\
104 \\
\text { Número de controles: } \\
103\end{array}$ & $\begin{array}{c}\text { Escore } \\
\text { total do FIQ } \\
\text { (Fibromyalgia } \\
\text { Impact } \\
\text { Questionnair) } \\
\\
\text { Escore do } \\
\text { componente } \\
\text { "dor" do FIQ }\end{array}$ & $\begin{array}{c}\text { - Limiar médio de dor nos tender } \\
\text { points } \\
\text { - Número de tender points } \\
\text { - Componente "fatiga" do FIQ } \\
\text { - Cansaço ao acordar } \\
\text { - Escore de rigidez } \\
\text { - Escore CGISS (Clinical Global } \\
\text { Impression of Improvement Scale) } \\
\text { - Escore PGIIS (Patient Global } \\
\text { Impression of Improvement Scale) } \\
\text { - Escore BPI (Brain Pain Inventory) } \\
\text { - Escore MOSSF-36 (Medical } \\
\text { Outcomes Study Short Form-36) } \\
\text { - Qualidade de vida na Escala de } \\
\text { Depressão } \\
\text { - Escala de Incapacidade de } \\
\text { Sheehan }\end{array}$ & $\begin{array}{c}\text { NNT: } 7,9 ; \\
\text { Intervalo de } \\
\text { confiança de } \\
95 \%(4,2-76,6) \\
\\
\text { Redução do risco } \\
\text { absoluto: } \\
12,59 \% ; \\
\text { Intervalo de } \\
\text { confiança de } \\
95 \%(1,31- \\
23,87 \%)\end{array}$ & \begin{tabular}{|c|}
3,11 \\
(análise do \\
impacto no \\
componente \\
"dor" do FIQ \\
escore \\
\\
- 3,04 (análise \\
da qualidade \\
de vida na \\
Escala de \\
Depressão)
\end{tabular} & $\begin{array}{l}\text { Apesar de obter um bom } \\
\text { valor do coeficinte "d" } \\
\text { de Cohen nas escalas que } \\
\text { envolviam depressão, o } \\
\text { estudo analisou a eficácia } \\
\text { da duloxetina em vários } \\
\text { sintomas (como citado nos } \\
\text { desfechos), e os resultados } \\
\text { mostraram que este } \\
\text { tratamento é eficaz para a } \\
\text { maioria deles, em pacientes } \\
\text { com ou sem depressão. } \\
\text { Comentários } \\
\text { Uma resposta efetiva } \\
\text { corresponde à redução } \\
\text { de pelo menos } 50 \% \text { no } \\
\text { componente "dor" do } \\
\text { escore FIQ. }\end{array}$ \\
\hline $\begin{array}{c}\text { Efficacy and safety of } \\
\text { milnacipran } 100 \mathrm{mg} / \\
\text { day in patients with } \\
\text { fibromyalgia: results } \\
\text { from a randomized, } \\
\text { double-bind, placebo- } \\
\text { controlled trial } \\
\text { Arthritis \& Reumatism } \\
2010 \\
\text { Arnold LM et al. } \\
\text { Número de patientes: } \\
516 \\
\text { Número de controles: } \\
509 \\
\end{array}$ & $\begin{array}{l}\text { Escore PGIC } \\
\text { (Patient's } \\
\text { Global } \\
\text { Impression of } \\
\text { Change) }\end{array}$ & $\begin{array}{c}\text { - Escore MOSSF-36 (Medical } \\
\text { Outcomes Study Short Form-36) } \\
\text { - Severidade da dor no BPI (Brain } \\
\text { Pain Inventory) } \\
\text { - Escore total do FIQ (Fibromyalgia } \\
\text { Impact Questionnair) } \\
\text { - Escore MFI (Multidimensional } \\
\text { Fatigue Inventory) }\end{array}$ & $\begin{array}{c}\text { NNT: } 6,3 ; \\
\text { Intervalo de } \\
\text { confiança de } \\
95 \%(4,2-76,6) \\
\text { Redução do risco } \\
\text { absoluto: } \\
15,93 \% ; \\
\text { Intervalo de } \\
\text { confiança de } \\
95 \%(10,22- \\
21,64 \%)\end{array}$ & $\begin{array}{c}-5,87 \text { (análise } \\
\text { do PGIC) } \\
1,06 \\
\text { (análise do } \\
\text { componente } \\
\text { mental do } \\
\text { MOSSF-36) }\end{array}$ & $\begin{array}{l}\text { A eficácia do milnacipran } \\
\text { na depressão não foi } \\
\text { avaliada baseada em escalas } \\
\text { apropriadas para distúrbios } \\
\text { psiquiátricos. Entretanto, } \\
\text { este fármaco mostrou-se } \\
\text { eficaz na melhora da dor, } \\
\text { fatiga, funções físicas e } \\
\text { mentais. }\end{array}$ \\
\hline $\begin{array}{c}\text { A randomized, } \\
\text { double-bind, placebo- } \\
\text { controlled study } \\
\text { of moclobemide } \\
\text { and amitriptyline } \\
\text { in the treatment of } \\
\text { fibromyalgia in females } \\
\text { without psychiatric } \\
\text { disorder } \\
\text { British Journal of } \\
\text { Rheumatology } \\
1998 \\
\text { Hannonen P et al. } \\
\text { Número de patientes: } \\
\text { (M) } 43 \\
\text { (A) } 42 \\
\text { Número de controles: } \\
45\end{array}$ & $\begin{array}{l}\text { Escore VAS } \\
\text { (Visual } \\
\text { Analogic } \\
\text { Scales) }\end{array}$ & $\begin{array}{c}\text { - Escore NHP (Nottingham Health } \\
\text { Profile) } \\
\text { - Escala de Incapacidade de } \\
\text { Sheehan }\end{array}$ & $\begin{array}{c}\text { Moclobemida } \\
\text { NNT: } 30,0 ; \\
\text { Intervalo de } \\
\text { confiança de } \\
95 \%(3,5-\infty) \\
\text { Redução do risco } \\
\text { absoluto: } \\
3,33 \% \text {; Intervalo } \\
\text { de confiança de } \\
95 \%(-21,96- \\
\text { +28,61) } \\
\text { Amitriptilina } \\
\text { NNT: } 4,0 ; \\
\text { Intervalo de } \\
\text { confiança de } \\
95 \%(3,5-\infty \text { ) } \\
\text { Redução do risco } \\
\text { absoluto: } \\
3,33 \% \text {; Intervalo } \\
\text { de confiança de } \\
95 \% \text { (1,65- } \\
48,35 \%)\end{array}$ & $\begin{array}{c}\text { Moclobemida } \\
0,15 \text { (análise } \\
\text { do VAS) } \\
-0,08 \\
\text { (componente } \\
\text { emocional do } \\
\text { NHP) } \\
\text { Amitriptilina } \\
\text { - 0,05 (análise } \\
\text { do VAS) } \\
-0,56 \\
\text { (componente } \\
\text { emocional do } \\
\text { NHP) }\end{array}$ & $\begin{array}{l}\text { O estudo concluiu que a } \\
\text { moclobemida não deve } \\
\text { ser utilizada no tratamento } \\
\text { da FM. Os pesquisadores } \\
\text { excluíram os pacientes } \\
\text { com depressão e, portanto, } \\
\text { o papel destas drogas em } \\
\text { desordens psiquiatrias } \\
\text { não pôde ser avaliado. No } \\
\text { entanto, a amitriptilina } \\
\text { mostrou um tamanho de } \\
\text { efeito moderado quando o } \\
\text { componente emocional foi } \\
\text { abordado. } \\
\text { Comentários } \\
\text { O estudo envolveu um } \\
\text { número pequeno de } \\
\text { participantes em cada } \\
\text { grupo, com uma alta taxa } \\
\text { de desistência durante o } \\
\text { seguimento completo. }\end{array}$ \\
\hline
\end{tabular}




\section{Estimativas}

- NNT = 1/RRA; onde RRA = redução do risco absoluto

- RRA = ITECI - ITEEI; quando TEC $=$ taxa de eventos de controle $/$ TEE $=$ taxa de eventos experimentais - Coeficiente "d" de Cohen:

$$
d=\frac{\bar{x}_{1}-\bar{x}_{2}}{s}, \cdots s_{1}^{2}=\frac{1}{n_{1}-1} \sum_{i=1}^{n_{1}}\left(x_{1, i}-\bar{x}_{1}\right)^{2} \quad \cdots \cdots=\sqrt{\frac{\left(n_{1}-1\right) s_{1}^{2}+\left(n_{2}-1\right) s_{2}^{2}}{n_{1}+n_{2}}},
$$

Quando: (x = média; s: desvio padrão)

\section{Conclusão}

Dessa forma, tornou-se evidente que a fibromialgia é ainda um desafio para os estudos médicos, uma vez que abrange inúmeros sintomas e comorbidades associados.

A fisiopatologia ainda permanece obscura, sendo descritos poucos avanços em pesquisas genéticas, além da ausência de exames diagnósticos específicos. O método clínico, por outro lado, permanece soberano, o único capaz de estabelecer o seu diagnóstico.

Como visto anteriormente, a concomitância de distúrbios psiquiátricos é bastante prevalente, e a depressão, conforme mostrada neste estudo, é um deles.

Por mais que inúmeras opções sejam consideradas no tratamento da fibromialgia, existem poucos estudos de evidência A-1, caracterizados por ensaios clínicos randomizados, duplo-cegos e controlados por placebo. E, ainda, alguns destes estudos apresentam vieses em sua execução.

Dados como NNT (number needed to treat) e tamanho de efeito são raramente encontrados nos artigos científicos que abordam a eficácia, não apenas dos antidepressivos na fibromialgia, mas das opções terapêuticas em geral. Quando, como leitores críticos, realizamos o cálculo dos mesmos, algumas surpresas são implacáveis, como NNTs elevados e tamanhos de efeito irrisórios, dentro de desfechos de pouca ou nenhuma significância clínica, como a melhora apenas parcial em algum escore predefinido e importância duvidosa para o paciente.

\section{Referências}

ARNOLD, L. M.; ClAUW, D. J.; McCABERG, B. H. Improving the recognition and diagnosis of fibromyalgia. Mayo Clin Proc, v.86, n.5, p. 457-464, 2011.

. et al. A double-bind, multicenter trial comparing duloxetine with placebo in the treatment of fibromyalgia patients with or without major depressive disorder. Arthritis Rheum, v.50, n.9, p. 2974-2984, 2004.

. et al. Efficacy and safety of milcacipran $100 \mathrm{mg} /$ day in patients with fibromyalgia: results of a randomized, double-bind, placebo-controlled trial. Arthritis Rheum, v.62, n.9, p. 2745-2756, 2010.

BERGLUND, B. et al. Quantitative and qualitative perceptual analysis of cold dysesthesia and hyperalgesia in fibromyalgia. Pain, v.96, n.1, p. 177-187, 2002.

BUSCH, A. J. et al. Exercise for treating fibromyalgia syndrome. Cochrane Database Sys Rev, I.4, 2007. DOI: 10.1002/14651858.CD003786.pub2.

CAVALCANTE, A. B. et al. A prevalência da fibromialgia: uma revisão da literatura. Rev Bras Reumatol, v.46, n.1, p. 40-48, 2006.

CEKO, M.; BUSHNELL, M. C.; GRACELY, R. H. Neurobiology underlying fibromyalgia symptoms. Pain Research and Treatment, v.2012, 2012 Article ID 585419, 8 pages. DOI: <http://dx.doi.org/10.1155/2012/585419>

CLAUW D. W.; ARNOLD, L. M.; McCARBERG, B. H. The science of fibromyalgia. Mayo Clin Proc, v.86, n.9, p. 907-911, 2011.

GEISSER, M. E. et al. Perception of noxious and innocuous heat stimulation among healthy women and women with fibromyalgia: association with mood, somatic focus, and catastrophizing. Pain, v.102, n.3, p. 243-250, 2003. 
GOLDENBERG, D. L. Multidisciplinary modalities in the treatment of fibromyalgia. J Clin Psychiatry, v. 69, v.2, p. 30-34, 2008.

GRACELY R. H. et al. Functional magnetic resonance imaging evidence of augmented pain processing in fibromyalgia. Arthritis Rheum, v.46, n.5, p. 1333-1343, 2002.

HÄUSER, W. et al. Treatment of fibromyalgia syndrome with antidepressants: a meta-analysis. JAMA, v.301, n.2, p. 108-209, 2009 .

HUDSON, J. I. et al. Fibromyalgia and major affective disorder: a controlled phenomenology and family history study. Am J Psychiatry, v.142, p.441-446, 1985.

KASSAM, A.; PATTEN, S. B. Major depression, fibromyalgia and labour force participation: a population based cross-sectional study. BMC Musculoskeletal Disord, v.7, n.4., 2006.

KÖLLNER, V. et al. Diagnosis and therapy on fibromyalgia syndrome. Psychoterp Psychosom Med Phychol, v.61, n.6, p. 276-285, 2011.

MARSON, P.; PASERO, G. Historical evolution of the concept of fibromyalgia: the main stages. Reumatismo, v.60, n.4, p.301-304, 2008

MARTINS, M. R. I. et al. Uso de questionários para avaliar a multidimensionalidade e a qualidade de vida do fibromiálgico. Rev Bras Reumatol, v.52, p. 16-26, 2012.

MATSUDA, J. B. et al. Polimorfismos dos genes do receptor da serotonina (5-HT2A) e da catecol-O-metiltransferase (COMT): fatores desencadeantes da fibromialgia? Rev Bras Reumatol, v.50, n.2, p. 141-149, 2010.

MERGENER, M. et al. Influência da interação entre qualidade ambiental e o SNPT102C do gene HTR2A sobre a susceptibilidade à fibromialgia. Rev Bras Reumatol, v.51, n.6, p. 587-602, 2011.

OKIFUJI, A. et al. A standardized manual tender point survey. Development and determination of a threshold point for the identification of positive tender points in fibromyalgia syndrome. J Rheumatol, v.24, n.2, p. 377-383, 1997.

RUSSELL, I. J.; RAPHAEL, K. G. Fibromyalgia syndrome: presentation, diagnosis, differential diagnosis, and vulnerability. CNS Spectr, v.13, n.3, p. 6-11, 2008.

SHUSTER, J. et al. Understanding the psychosocial profile of women with fibromyalgia syndrome. Pain Res Manaq, v.14, n.3, p.239-245, 2009.

SMITH, B. et al. Drug class review: drugs for fibromyalgia. Final Original Report. Portland (OR): Oregon Health \& Science University, 2011.

SMITH, H.S.; HARRIS, R.; CLAW, D. Fibromyalgia: an afferent processing disorder leading to a complex pain generalized syndrome. Pain Physician, v. 14, n.2, p. 217245,2011

STAUD, R. et al. Abnormal sensitization and temporal summation of second pain (wind-up) in patients with fibromyalgia syndrome. Pain, v.91, n.1, p.165-175, 2001

THIEME, K. et al. Psychological pain treatment in fibromyalgia syndrome: efficacy of operant behavioral and cognitive behavioral treatments. Arthritis Res Ther, v.8, n.4, p.121, 2006

WOLFE, F. et al. The prevalence and characteristics of fibromyalgia in the general population. Arthritis Rheum, v.38, p. 19-28, 1995.

et al. The American College of Rheumatology 1990. Criteria for the classification of fibromyalgia. Report of the Multicenter Criteria Committee. Arthritis Rheum, v.33, n.2, p. 160-172, 1990.

WOLFE, F. et al. Fibromyalgia criteria and severity scales for clinical and epidemiological studies: a modification of the ACRE preliminary diagnostic criteria for fibromyalgia. J Rheumatol, v.38, n.6, p. 1113-1122, 2011.

WOOD, P. B.; SCHWEINHARDT, P.; JAEGER, E. Fibromyalgia patients show an abnormal dopamine response to pain. Eur J Neurosci, v.25, n.12, p. 3576-3582, 2007.

YUNUS, M. B. Fibromyalgia and overlapping disorders: the unifying concept of central sensivity syndromes. Semin Arthritis Rheum, v.36, n.6, p. 339-356, 2007.

Role of central sensibilization in symptoms beyond muscle pain, and the evaluation of a patient with widespread pain. Best Pract Res Clin Rheumatol, v.21, n.3, p. 481497, 2007. 